\title{
ALIMENTAÇÃO DOS PEIXES EM UM RIACHO DO PARQUE ESTADUAL MORRO DO DIABO, BACIA DO ALTO RIO PARANÁ, SUDESTE DO BRASIL
}

\author{
Lilian Casatti
}

\author{
Biota Neotropica v2 (n2) - http://www.biotaneotropica.org.br/v2n2/pt/abstract?article+BN02502022002 \\ Date Received 09/02/2002 \\ Revised 11/14/2002 \\ Accepted 11/23/2002
}
Laboratório de Ictiologia, Departamento de Zoologia e Botânica, IBILCE, Universidade Estadual Paulista (www.ibilce.unesp.br), Rua Cristóvão Colombo, 2265, 15054-000, São José do Rio Preto, SP, Brasil
(e-mail: licasatti@hotmail.com)

\begin{abstract}
The trophic structure of a fish assemblage in a first order stream in the upper Paraná River basin was investigated using standard methods of diet analysis and underwater observations utilizing snorkeling. Three stretches of the Córrego São Carlos were studied. Eighteen fish species belonging to five orders and ten families were captured. The stomach analysis of 299 fishes revealed that $70 \%$ of the food items are autochthonous, $24 \%$ allochthonous, and $6 \%$ material of unidentifiable origin. Eighteen pairs of species (33\%) showed significant feeding overlap, though this overlap does not necessarily indicate competition given the temporal and spatial segregation during foraging. Three feeding guilds were found. The invertivores included Astyanax altiparanae, Moenkhausia sanctaefilomenae, and Oligosarcus pintoi, whose diet demonstrated a predominance of allochthonous items, and Rhamdia quelen, Trichomycterus sp., Corydoras aeneus, and Crenicichla britskii, that had a predominance of autochthonous items in their diets. In this group, A. altiparanae and $M$. sanctaefilomenae are drift feeders, $R$. quelen is a benthic opportunistic predator, Trichomycterus sp. and C. aeneus are grubbers, and $O$. pintoi and $C$. britskii are ambush predators. The omnivore with a tendency to herbivory is represented by Phalloceros caudimaculatus, which feeds mainly on algae. The periphitivores included Hisonotus sp., Hypostomus nigromaculatus, and $H$. ancistroides that are grazers with a diet composed mostly of diatoms, clorophyts, and organic matter. The results indicated that the fish assemblage in the Córrego São Carlos is structured at spatial, temporal, and trophic levels, and shows partitioning of the food resources. The addition of the fish species in each trophic guild along the stream is possibly due to the longitudinal increase of microhabitats that makes available more feeding sites.
\end{abstract}

Key words: stream fishes, feeding, spatial segregation, trophic segregation, resource partitioning

Resumo - Neste estudo foi investigada a estrutura trófica de uma comunidade de peixes de um riacho de primeira ordem na bacia do Alto Rio Paraná, empregando métodos habituais de análise da dieta combinados com observações naturalísticas. Três trechos do Córrego São Carlos foram estudados. Foram coletadas 18 espécies de peixes, pertencentes a cinco ordens e dez famílias. A análise de 299 estômagos mostrou que 70\% dos itens alimentares são autóctones, 24\% alóctones e 6\% material de origem não identificada. Dezoito pares de espécies (33\%) apresentaram sobreposição alimentar significativa, porém esta sobreposição não necessariamente indica competição em razão da segregação espacial e temporal observada na captura do alimento. Três guildas alimentares foram determinadas. Os invertívoros incluíram Astyanax altiparanae, Moenkhausia sanctaefilomenae e Oligosarcus pintoi, que apresentaram predominância de itens alóctones, e Rhamdia quelen, Trichomycterus sp., Corydoras aeneus e Crenicichla britskii, com predominância de itens autóctones. Neste grupo A. altiparanae e $M$. sanctaefilomenae são catadores de itens na coluna d'água, $R$. quelen é um predador oportunista bentônico, Trichomycterus sp. e C. aeneus são especuladores de substrato, O. pintoi e C. britskii são predadores de emboscada. Os onívoros com tendência à herbivoria foram representados por Phalloceros caudimaculatus, que se alimentou principalmente de algas. Os perifitívoros incluíram Hisonotus sp., Hypostomus nigromaculatus e Hypostomus ancistroides, pastadores com dieta composta principalmente por diatomáceas, clorofíceas e matéria orgânica. Os resultados aqui encontrados indicam que a comunidade de peixes no Córrego São Carlos se mostra estruturada em nível espacial, temporal e trófico, apresentando uso partilhado dos recursos alimentares disponíveis. O acréscimo de espécies em cada categoria trófica ao longo do riacho possivelmente é um reflexo da crescente heterogeneidade longitudinal de microhábitats na área, disponibilizando sítios de alimentação adicionais.

Palavras-chave: peixes de riacho, alimentação, segregação espacial, segregação trófica, partilha de recursos 


\section{Introdução}

Historicamente, o conhecimento da alimentação de peixes de riachos, incluindo o uso de recursos e a influência dos componentes espaciais e temporais, tem subsidiado estudos sobre estruturação dessas comunidades e contribuído para a investigação de interações biológicas, tais como predação e competição (Esteves \& Aranha, 1999). Nos últimos 20 anos, esse conhecimento tem sido aplicado mais diretamente na avaliação da integridade biótica de riachos, sendo utilizado como fonte de atributos para cálculos de índices de integridade biótica (Karr, 1981; Smogor \& Angermeier, 1999). Para a aplicação correta destes índices é necessário conhecer a condição natural examinada nos chamados "riachos-referência”, ou seja, aqueles que apresentam o mínimo de influência antrópica possível (Hughes, 1995).

É bem sabido que os recursos aquáticos do Estado de São Paulo encontram-se seriamente impactados e, por esta razão, possíveis riachos-referência são quase que exclusivamente encontrados em áreas de conservação (vide Inventário Florestal do Estado de São Paulo, 2000), das quais o Parque Estadual Morro do Diabo se destaca por incluir a maior área de proteção ambiental (aproximadamente 34 mil hectares) da drenagem do Alto Rio Paraná no Estado de São Paulo (Clauset, 1999). Até recentemente a ictiofauna desse Parque nunca havia sido estudada. A presente autora e colaboradores estudaram quatro riachos do Parque Estadual Morro do Diabo, onde registraram 22 espécies de peixes (Casatti et al., 2001). Um destes riachos, o Córrego São Carlos, foi investigado com maior detalhe em razão de ser o mais adequado para a realização de observações subaquáticas através de mergulho livre, uma abordagem especialmente interessante na obtenção de informações naturalísticas utilizadas na interpretação da alimentação da comunidade de peixes estudada (Sabino, 1999). O objetivo do presente estudo foi investigar a estrutura trófica dos peixes do Córrego São Carlos, um riacho-referência de primeira ordem na bacia do Alto Rio Paraná, empregando métodos habituais de análise da dieta combinados com observações naturalísticas.

\section{Local de estudo}

O Córrego São Carlos é um riacho de primeira ordem (1:50.000), com extensão aproximada de $5 \mathrm{~km}$, afluente direto do Rio Paranapanema e corre em uma área de vegetação nativa caracterizada como Floresta Estacional Semi-decídua, pertencente ao Parque Estadual Morro do Diabo (Fig. 1), município de Teodoro Sampaio, Estado de São Paulo. O clima é tropical subquente úmido (Nimer, 1989) com uma estação seca, de abril a setembro (menor precipitação de 14,7 mm em maio 2000), e outra chuvosa, de outubro a março (maior precipitação de $361 \mathrm{~mm}$ em dezembro 2000). Três trechos eqüidistantes (100 m de extensão cada) e representativos das porções superior, média e inferior foram escolhidos para a realização das observações subaquáticas e coleta dos peixes (Tabela 1).

Ao longo do riacho as margens são suavemente sinuosas, com trechos apresentando planícies cobertas por gramíneas (principalmente Commelinaceae e Poaceae) e pteridófitas (Pteridaceae, Polypodiaceae e Sellaginellaceae). Durante o período de estudo, a transparência horizontal da água variou de 2 a 2,75 m, a temperatura da água de 19,2 a $21,1^{\circ} \mathrm{C}$, o pH de 6,81 a 7,98, a condutividade de 14,7 a 16,2 $\mu \mathrm{S} / \mathrm{cm}$ e o oxigênio dissolvido de 8,8 a 10,9 mg/l.

\section{Material e métodos}

O trabalho de campo foi conduzido de junho de 2000 a março de 2001 em quatro viagens, regularmente distribuídas a cada três meses. Uma viagem preliminar foi realizada em março de 2000. Um total de 17 horas (13 horas diurnas e 4 noturnas) de mergulho livre foi realizado nos trechos médio e inferior, utilizando os métodos animal focal e "ad libitum" (Lehner, 1998), durante as quais foram registradas as seguintes variáveis: número de indivíduos de cada espécie, sua posição na coluna d’água, tipo de fundo, tática de forrageamento (cf. Curio, 1976; Keenleyside, 1979; Sazima, 1986 e Grant \& Noakes, 1987), período e local de forrageamento. No trecho superior não foram realizadas observações subaquáticas em razão da pequena profundidade (máximo de $30 \mathrm{~cm}$ ). O término do pôr-do-Sol foi considerado o limite entre dia e noite. Indivíduos avistados fora de abrigos (nadando, procurando alimento ou alimentando-se) foram considerados ativos, enquanto que aqueles abrigados ou estacionários durante a maior parte do período de observação foram considerados inativos (Gibran \& Castro, 1999).

Na captura dos peixes foi empregado um esforço padronizado de coleta em cada viagem, que consistiu em utilizar duas peneiras (70 cm de diâmetro, 2,5 mm entre-nós) e uma rede de arrasto manual (2,5 mm entre-nós) por aproximadamente 40 minutos cada. Os peixes foram preservados em formalina a $10 \%$ e, após a fixação, transferidos para etanol 70\%. As espécies consideradas residentes foram aquelas presentes em pelo menos $50 \%$ das coletas (Dajoz, 1983, modificado). A análise dos conteúdos estomacais seguiu Knöppel (1970). Para cada item alimentar foram calculadas a freqüência de ocorrência (Bowen, 1992) e a composição percentual (Hynes, 1950). O termo perifitívoro segue a definição de Uieda et al. (1997). O índice de sobreposição alimentar adotado foi o de Morisita modificado por Horn (1966), calculado com os valores de composição percentual dos itens alimentares agrupados em categorias amplas. Os valores considerados significativos foram aqueles $\geq 0,58$ (Linton et al., 1981).

A similaridade entre a dieta das espécies estudadas foi calculada a partir dos valores de composição percentual 


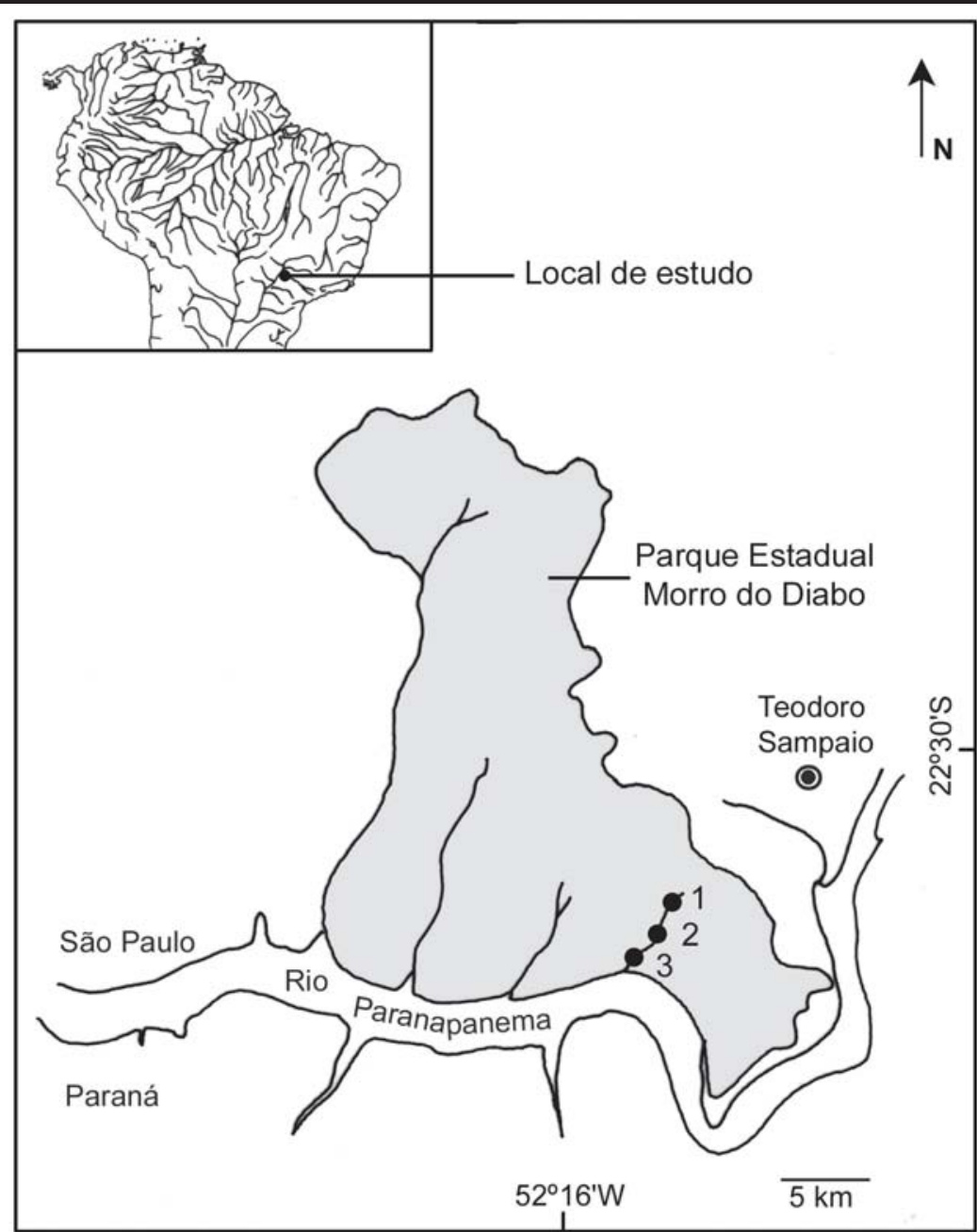

Figura 1. Localização do Córrego São Carlos, Parque Estadual Morro do Diabo, Estado de São Paulo, indicando os trechos superior (1), médio (2) e inferior (3).

\begin{tabular}{lccc}
\hline Parâmetros & Superior & Médio & Inferior \\
\hline Coordenadas & $22^{\circ} 35^{\prime} 28.0^{\prime \prime} \mathrm{S}$ & $22^{\circ} 35^{\prime} 54.4^{\prime \prime} \mathrm{S}$ & $22^{\circ} 36^{\prime} 23.8^{\prime \prime} \mathrm{S}$ \\
& $52^{\circ} 14^{\prime} 38.1^{\prime \prime} \mathrm{W}$ & $52^{\circ} 14^{\prime} 45.2^{\prime \prime} \mathrm{W}$ & $52^{\circ} 15^{\prime} 08.6^{\prime \prime} \mathrm{W}$ \\
Altitude (m) & 294 & 286 & 284 \\
$\begin{array}{l}\text { Velocidade média da corrente } \\
(\mathrm{m} / \mathrm{s})\end{array}$ & 1,5 & 0,3 & 0,6 \\
$\begin{array}{l}\text { Composição predominante do } \\
\text { fundo }\end{array}$ & $\begin{array}{c}\text { rochas, seixos e } \\
\text { cascalhos }\end{array}$ & $\begin{array}{c}\text { areia, com seixos em } \\
\text { pequenas corredeiras }\end{array}$ & $\begin{array}{c}\text { areia, com troncos, } \\
\text { galhadas e folhiço }\end{array}$ \\
Variação de largura (m) & $0,8-2,6$ & $0,9-3,6$ & $1,3-3,9$ \\
Profundidade máxima (m) & 0,3 & 0,5 & 0,9 \\
\hline
\end{tabular}

Tabela 1. Caracterização dos trechos estudados no Córrego São Carlos, Parque Estadual Morro do Diabo, sudeste do Brasil. 
através do método de aglomeração por ligação simples usando o coeficiente de Bray-Curtis (cf.Valentin, 1995), sendo o resultado exibido na forma de dendrograma. Este cálculo foi processado com auxílio do programa de computador BioDiversity-PRO (McAlecee et al., 1997).

\section{Resultados}

\subsection{Ictiofauna}

Foram coletadas 18 espécies de peixes no Córrego São Carlos, pertencentes a cinco ordens e dez famílias, num total de 940 indivíduos e 1.671 g de biomassa. Do total de espécies coletadas, 11 foram consideradas residentes (Tabela 2), das quais as mais abundantes foram Phalloceros caudimaculatus, Hypostomus nigromaculatus, Hisonotus sp., Trichomycterus sp. e H. ancistroides. No trecho superior as espécies mais abundantes foram Trichomycterus sp. e H. nigromaculatus; no médio foram $P$. caudimaculatus e $H$. nigromaculatus; no inferior foram Hisonotus sp. e $P$. caudimaculatus (vide Tabela 2).

\subsection{Comportamento alimentar e dieta}

Dezesseis espécies de peixes foram observadas durante mergulho, para as quais foram registradas sete táticas alimentares (Tabela 3). A análise de 299 estômagos das 11 espécies residentes (Tabelas 4 e 5) mostrou que $70 \%$ dos itens alimentares são autóctones, 24 \% alóctones e 6\% material de origem não identificada (Figura 2). Algas (diatomáceas e clorofíceas), fragmentos de vegetais superiores, larvas de insetos aquáticos (Diptera e Trichoptera) e bivalves representaram 86\% dos itens de origem autóctone, enquanto que Hymenoptera (Formicidae) representou $61 \%$ dos itens de origem alóctone. Não foram encontradas diferenças sazonais na ocorrência dos itens alimentares.

De acordo com a similaridade entre a composição percentual das dietas das espécies analisadas três guildas alimentares foram estabelecidas (Figura 3). Não foi possível realizar uma análise comparativa da dieta dos peixes entre os trechos amostrados, pois algumas espécies foram numericamente pouco representadas. Ao longo do riacho as três guildas alimentares foram representadas, variando apenas em sua composição (Tabela 6). Dezoito pares de espécies (33\% dos casos de sobreposição) apresentaram sobreposição alimentar significativa (Tabela 7).

\subsubsection{Invertívoros}

Neste grupo foram incluídas sete espécies de peixes que ingeriram principalmente insetos. No dendrograma de similaridade entre as dietas (Figura 3) nota-se também maior semelhança entre as espécies de acordo com a origem do alimento. Os caracídeos se alimentam principalmente de itens alóctones (Formicidae) enquanto que os siluriformes e o ciclídeo se alimentam principalmente de itens autóctones (larvas aquáticas de Diptera e Trichoptera).

Os lambaris, todos de hábito alimentar diurno, apresentaram elevada sobreposição alimentar. Oligosarcus pintoi é um predador de emboscada ("ambush predator”, cf. Sazima, 1986) enquanto que $M$. sanctaefilomenae e $A$. altiparanae praticam com mais freqüência (95\% dos registros) a cata de itens arrastados pela corrente ("driftfeeding”, cf. Grant \& Noakes, 1987). Oligosarcus pintoi permanece nadando lentamente em poços mais profundos junto de remansos marginais e, quando percebe alguma partícula alimentar, desloca-se rapidamente em um único impulso para abocá-la. Astyanax altiparanae forma grupos de até 30 indivíduos que nadam à meia-água coletando partículas arrastadas pela corrente; em algumas ocasiões foram avistados investindo contra raízes submersas de gramíneas marginais. Durante a noite, os indivíduos de $A$. altiparanae permanecem estacionários nos poços mais profundos do riacho; porém, em duas ocasiões no período noturno, adultos dormentes foram observados capturando insetos que submergiam na água, vindos da vegetação ripária. Moenkhausia sanctaefilomenae, formando grupos de três a quatro indivíduos, foi avistada ocupando posições marginais dos cardumes de A. altiparanae.

Rhamdia quelen, Trichomycterus sp. e C. aeneus, também com alta sobreposição alimentar entre si, capturam alimento junto ao fundo do riacho. A primeira espécie forrageia a partir do anoitecer, entre rochas, sem revolver o substrato ("crepuscular-nocturnal bottom predator", cf. Sazima, 1986), enquanto que Trichomycterus sp. e C. aeneus alimentam-se durante o dia. Neste processo revolvem superficialmente o substrato à procura de presas que são abocadas rapidamente através de sucção ("hunting speculation" cf. Curio, 1976 e "grubbers excavating while moving”, cf. Sazima, 1986). Crenicichla britskii apresentou sobreposição alimentar com a maioria das espécies invertívoras, com os maiores valores observados para $R$. quelen, Trichomycterus sp. e C. aeneus. Durante o dia ocupa principalmente abrigos junto de raízes submersas da vegetação marginal, apresenta-se semi-estacionária, com coloração disruptiva e captura suas presas através da tática de emboscada.

\subsubsection{Onívoro com tendência à herbivoria}

Os itens ingeridos por Phalloceros caudimaculatus foram principalmente de origem autóctone. Algas e fragmentos de vegetais superiores corresponderam a 71\% da dieta, contra 29\% representados por microcrustáceos (Copepoda e Cladocera) e insetos aquáticos, o que justifica a classificação de $P$. caudimaculatus nesta guilda alimentar. A elevada participação de algas e vegetais superiores na 


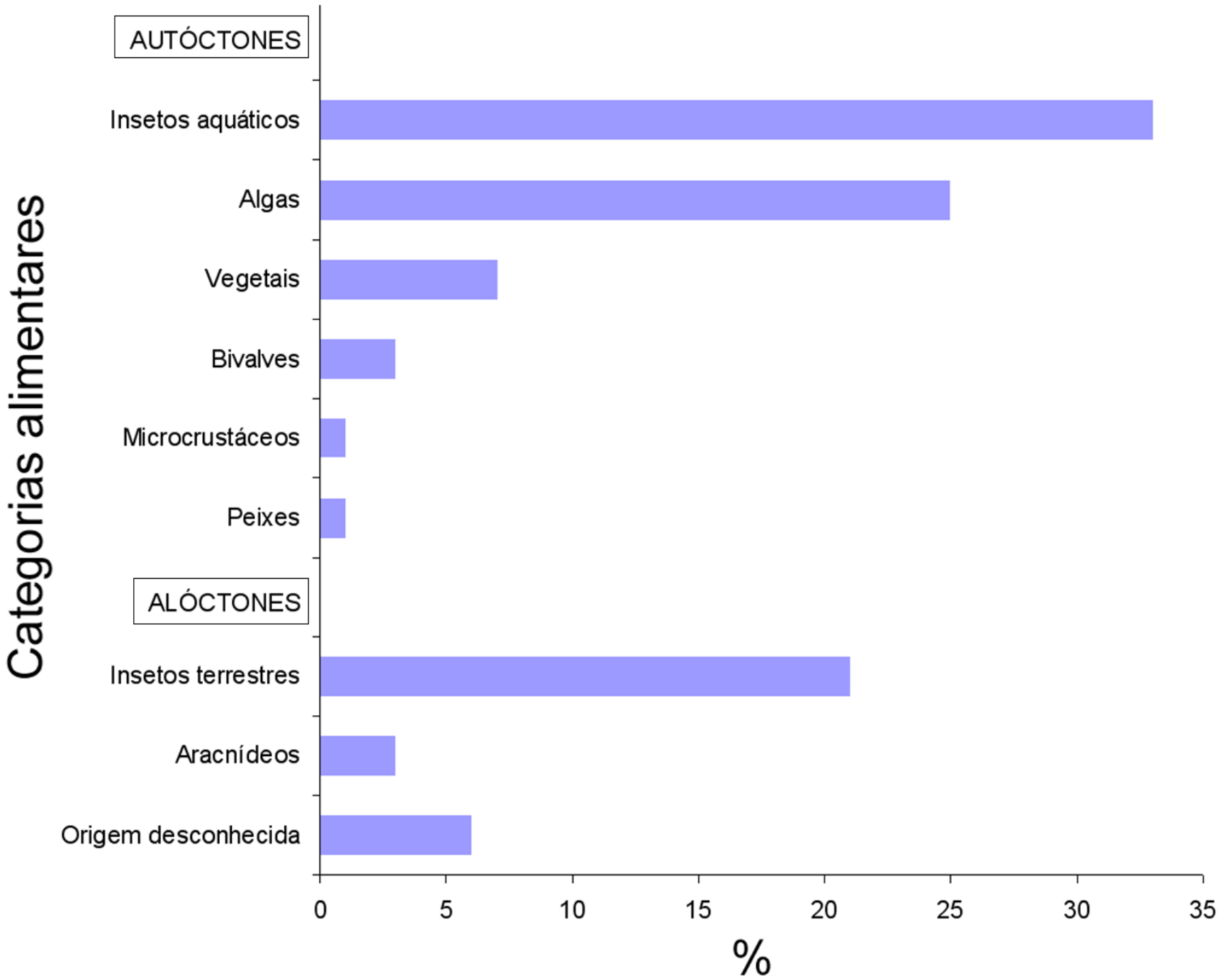

Figura 2. Diagrama de barras da composição percentual das dietas combinada de 11 espécies de peixes no Córrego São Carlos com os itens alimentares agrupados em categorias ecológicas amplas. 


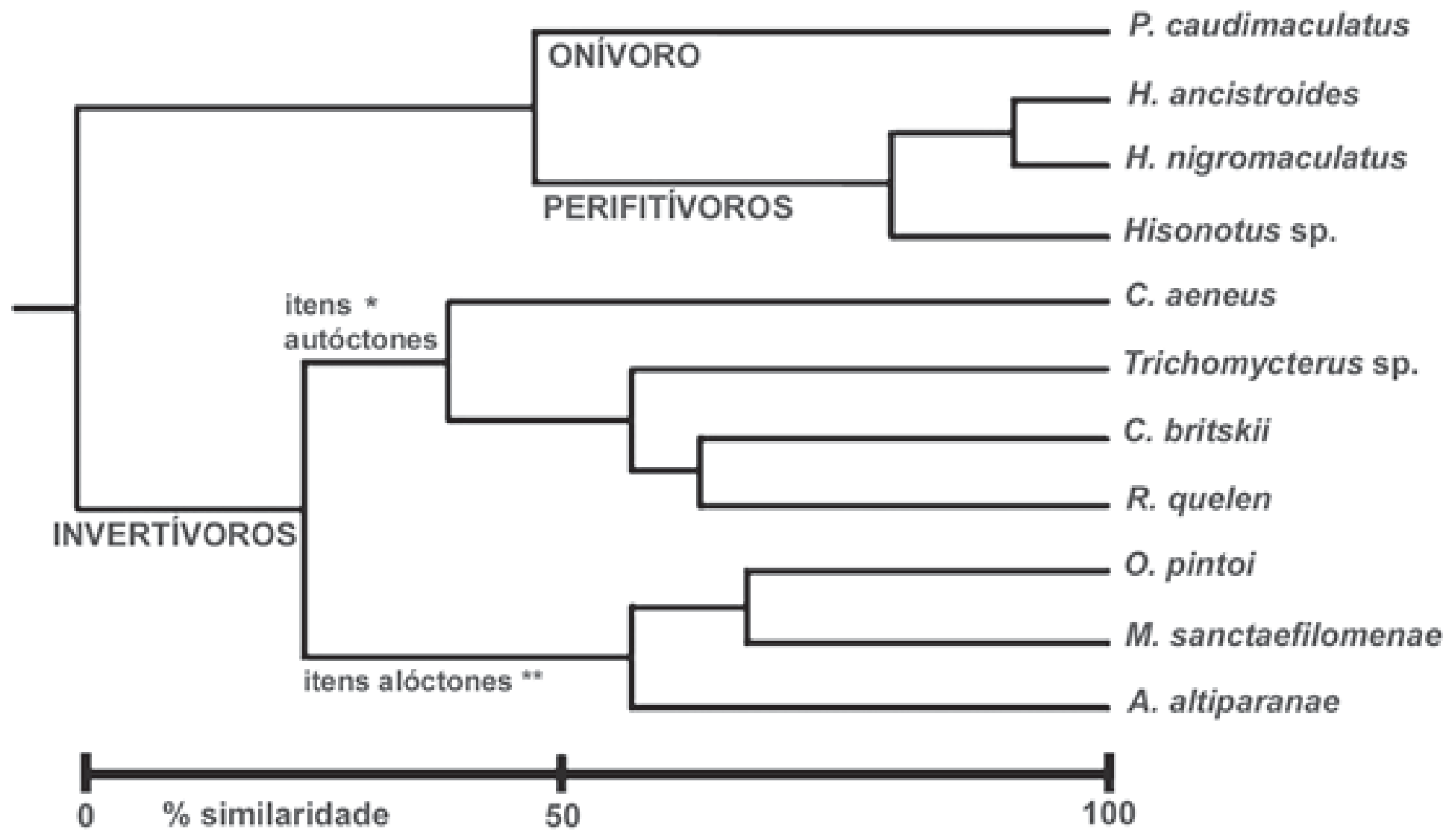

Figura 3. Dendrograma de similaridade sobre os valores de composição percentual da dieta de 11 espécies de peixes no Córrego São Carlos. * Predominância de larvas aquáticas de insetos, ** predominância de insetos terrestres. 


\begin{tabular}{|c|c|c|c|c|}
\hline Espécies por trecho & $\begin{array}{c}\text { Constância de } \\
\text { ocorrência }\end{array}$ & Estação seca & Estação chuvosa & Total \\
\hline \multicolumn{5}{|l|}{ Superior } \\
\hline Rhamdia quelen & 50 & - & 4 & 4 \\
\hline Trichomycterus sp. & 100 & 36 & 71 & 107 \\
\hline Hisonotus sp. & 25 & 2 & - & 2 \\
\hline Hypostomus ancistroides & 50 & 1 & 1 & 2 \\
\hline Hypostomus nigromaculatus & 100 & 76 & 87 & 163 \\
\hline Phalloceros caudimaculatus & $\begin{array}{l}100 \\
\text { Subtotal }\end{array}$ & $\begin{array}{c}12 \\
127\end{array}$ & $\begin{array}{c}21 \\
184\end{array}$ & $\begin{array}{c}33 \\
311\end{array}$ \\
\hline \multicolumn{5}{|l|}{ Médio } \\
\hline Oligosarcus pintoi & 25 & - & 2 & 2 \\
\hline Rhamdia quelen & 75 & 7 & 2 & 9 \\
\hline Trichomycterus sp. & 100 & 5 & 15 & 20 \\
\hline Hisonotus sp. & 100 & 13 & 10 & 23 \\
\hline Hypostomus ancistroides & 100 & 7 & 14 & 21 \\
\hline Hypostomus nigromaculatus & 100 & 11 & 21 & 32 \\
\hline Corydoras aeneus & 50 & 1 & 1 & 2 \\
\hline Phalloceros caudimaculatus & 100 & 39 & 96 & 135 \\
\hline \multirow[t]{2}{*}{ Synbranchus marmoratus } & 25 & - & 1 & 1 \\
\hline & Subtotal & 83 & 162 & 245 \\
\hline \multicolumn{5}{|l|}{ Inferior } \\
\hline Hoplias malabaricus & 25 & - & 1 & 1 \\
\hline Astyanax altiparanae & 75 & 4 & 3 & 7 \\
\hline Astyanax sp. & 25 & 1 & - & 1 \\
\hline Moenkhausia sanctaefilomenae & 100 & 7 & 7 & 14 \\
\hline Oligosarcus pintoi & 100 & 12 & 5 & 17 \\
\hline Characidium sp. & 25 & - & 1 & 1 \\
\hline Imparfinis mirini & 25 & 2 & - & 2 \\
\hline Phenacorhamdia hohenei & 25 & - & 1 & 1 \\
\hline Rhamdia quelen & 50 & 3 & 1 & 4 \\
\hline Pimelodella aff. gracilis & 25 & 1 & - & 1 \\
\hline Hisonotus sp. & 100 & 79 & 62 & 141 \\
\hline Hypostomus ancistroides & 100 & 29 & 36 & 65 \\
\hline Hypostomus nigromaculatus & 100 & 4 & 6 & 10 \\
\hline Corydoras aeneus & 100 & 7 & 10 & 17 \\
\hline Phalloceros caudimaculatus & 100 & 11 & 81 & 92 \\
\hline \multirow[t]{3}{*}{ Crenicichla britskii } & 75 & 2 & 8 & 10 \\
\hline & Subtotal & 162 & 222 & 384 \\
\hline & Total & 372 & 568 & 940 \\
\hline
\end{tabular}

Tabela 2. Espécies de peixes, constância de ocorrência (\%) e número de exemplares coletados nos trechos estudados do Córrego São Carlos, Parque Estadual Morro do Diabo, sudeste do Brasil, nas estações seca e chuvosa. 


\begin{tabular}{|c|c|c|c|}
\hline Táticas alimentares & Espécies & Períodos & Micro-hábitats \\
\hline \multirow[t]{3}{*}{$\begin{array}{l}\text { Predadores } \\
\text { de emboscada }\end{array}$} & Hoplias malabaricus* & $\begin{array}{l}\text { crepúsculo } \\
\text { vespertino }\end{array}$ & $\begin{array}{l}\text { água, entre raízes submersas da } \\
\text { vegetação marginal }\end{array}$ \\
\hline & Oligosarcus pintoi & dia & $\begin{array}{l}\text { meia água, em pequenos poços junto } \\
\text { às margens }\end{array}$ \\
\hline & Crenicichla britskii & dia & $\begin{array}{l}\text { meia água, entre raízes submersas da } \\
\text { vegetação marginal }\end{array}$ \\
\hline \multirow{3}{*}{$\begin{array}{l}\text { Catadores de itens } \\
\text { arrastados pela corrente }\end{array}$} & Astyanax altiparanae & dia & meia água, no meio do canal \\
\hline & Astyanax sp. * & dia & meia água, junto às margens \\
\hline & Moenkhausia sanctaefilomenae & dia & $\begin{array}{l}\text { meia água, junto às margens e } \\
\text { também entre agrupamentos de } A \text {. } \\
\text { altiparanae }\end{array}$ \\
\hline $\begin{array}{l}\text { Cata de itens } \\
\text { na superfície }\end{array}$ & Phalloceros caudimaculatus & dia & $\begin{array}{l}\text { na superfície, em poços rasos junto } \\
\text { às margens }\end{array}$ \\
\hline Predador de espreita & Characidium sp. * & dia & no fundo, entre rochas \\
\hline \multirow{4}{*}{$\begin{array}{l}\text { Especuladores } \\
\text { de substrato }\end{array}$} & Trichomycterus sp. & dia & no fundo, entre rochas \\
\hline & Corydoras aeneus & dia & $\begin{array}{l}\text { no fundo arenoso em pequenos } \\
\text { poços junto às margens }\end{array}$ \\
\hline & Imparfinis mirini ${ }^{*}$ & noite & no fundo, entre rochas \\
\hline & Phenacorhamdia hohenei * & noite & $\begin{array}{l}\text { no fundo, entre rochas e entre raízes } \\
\text { submersas da vegetação marginal }\end{array}$ \\
\hline $\begin{array}{l}\text { Predador } \\
\text { bentônico } \\
\text { oportunista }\end{array}$ & Rhamdia quelen & $\begin{array}{l}\text { crepúsculo } \\
\text { vespertino e noite }\end{array}$ & $\begin{array}{l}\text { no fundo, em poços mais } \\
\text { profundos }\end{array}$ \\
\hline \multirow[t]{3}{*}{ Pastadores } & Hisonotus sp. & dia & $\begin{array}{l}\text { pousados sobre a vegetação } \\
\text { marginal submersa }\end{array}$ \\
\hline & Hypostomus ancistroides & noite & $\begin{array}{l}\text { pousados sobre rochas junto ao } \\
\text { fundo ou sobre galhadas submersas }\end{array}$ \\
\hline & Hypostomus nigromaculatus & $\begin{array}{l}\text { crepúsculo } \\
\text { vespertino e noite }\end{array}$ & $\begin{array}{l}\text { pousados sobre rochas } \\
\text { junto ao fundo }\end{array}$ \\
\hline
\end{tabular}

Tabela 3. Táticas alimentares, períodos do dia e micro-hábitats preferenciais de forrageamento de 16 espécies observadas durante mergulhos no Córrego São Carlos, Parque Estadual Morro do Diabo, sudeste do Brasil. Asteriscos (*) indicam espécies ocasionais. 


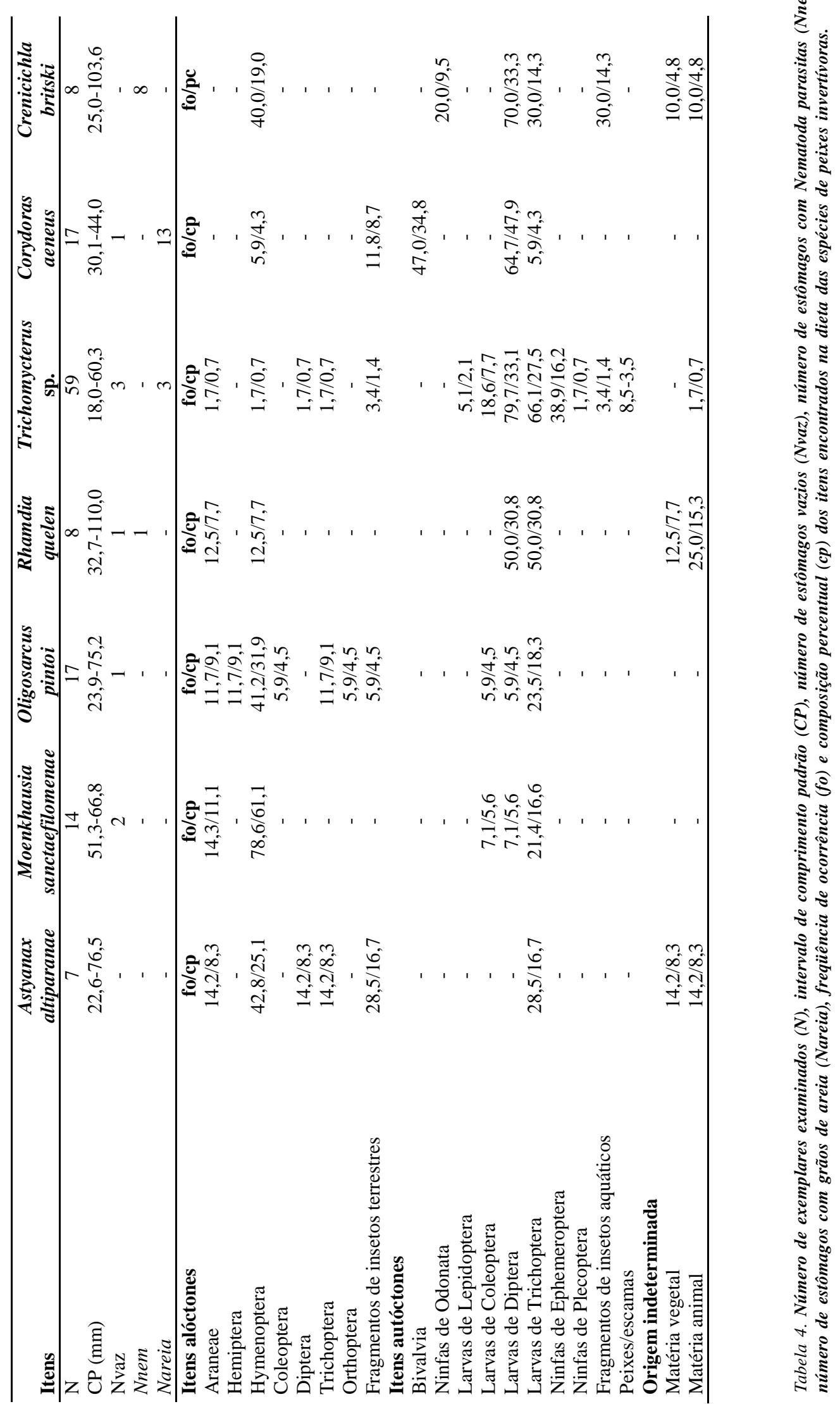




\begin{tabular}{lcccc}
\hline Itens & $\begin{array}{c}\text { Phalloceros } \\
\text { caudimaculatus }\end{array}$ & $\begin{array}{c}\text { Hisonotus } \\
\text { sp. }\end{array}$ & $\begin{array}{c}\text { Hypostomus } \\
\text { ancistroides }\end{array}$ & $\begin{array}{c}\text { Hypostomus } \\
\text { nigromaculatus }\end{array}$ \\
\hline $\mathrm{N}$ & 40 & 40 & 39 & 40 \\
$\mathrm{CP}(\mathrm{mm})$ & $14,4-28,9$ & $17,9-36,7$ & $28,3-85,2$ & $18,9-65,5$ \\
$\mathrm{Nvaz}$ & - & - & - & - \\
Nnem & - & - & - & - \\
Nareia & 12 & 20 & 25 & 21 \\
\hline Itens autóctones & $\mathbf{f o / c p}$ & $\mathbf{f o / c p}$ & $\mathbf{f o / c p}$ & $\mathbf{f o / c p}$ \\
Euglenophyceae & $22,5 / 6,1$ & - & - & $2,5 / 1,0$ \\
Cyanophyceae (filamentosa) & $57,5 / 15,4$ & $2,5 / 1,0$ & - & $5,0 / 2,0$ \\
Bacillariophyceae & $82,5 / 22,1$ & $100,0 / 43,0$ & $100,0 / 47,5$ & $97,5 / 39,4$ \\
Chlorophyta & $32,5 / 8,7$ & $45,0 / 20,0$ & $74,3 / 35,4$ & $87,5 / 35,3$ \\
Copepoda & $22,5 / 6,1$ & - & - & - \\
Cladocera & $12,5 / 3,3$ & - & - & - \\
Diptera & $37,5 / 10,1$ & - & - & - \\
Trichoptera & $2,5 / 0,7$ & - & - & - \\
Fragmentos de insetos aquáticos & $35,0 / 9,4$ & - & - & - \\
Escamas & $10,0 / 2,7$ & - & - & - \\
Origem indeterminada & & & & - \\
Fragmentos de vegetais superiores & $57,5 / 15,4$ & $45,0 / 20,0$ & - & - \\
Matéria orgânica & - & $37,5 / 16,0$ & $35,9 / 17,1$ & $55,0 / 22,3$ \\
\hline
\end{tabular}

Tabela 5. Número de exemplares examinados ( $N)$, intervalo de comprimento padrão (CP), número de estômagos vazios (Nvaz), número de estômagos com Nematoda parasitas (Nnem), número de estômagos com grãos de areia (Nareia), freqüência de ocorrência (fo) e composição percentual (cp) dos itens encontrados na dieta das espécies de peixes onívoras e perifitívoras.

\begin{tabular}{|c|c|c|c|}
\hline Guildas & Superior & Médio & Inferior \\
\hline Onívoros & Phalloceros caudimaculatus (33) & Phalloceros caudimaculatus (135) & Phalloceros caudimaculatus (92) \\
\hline \multirow[t]{3}{*}{ Perifitívoros } & Hypostomus nigromaculatus (163) & Hypostomus nigromaculatus (32) & Hisonotus sp. (141) \\
\hline & Hisonotus sp. (2) & Hisonotus sp. (23) & Hypostomus ancistroides (65) \\
\hline & Hypostomus ancistroides (2) & Hypostomus ancistroides (21) & Hypostomus nigromaculatus (10) \\
\hline \multirow[t]{6}{*}{ Invertívoros } & Trichomycterus sp. (107) & Trichomycterus sp. (20) & Oligosarcus pintoi (17) \\
\hline & & Rhamdia quelen (9) & Moenkhausia sanctaefilomenae (14) \\
\hline & & Oligosarcus pintoi (2) & Corydoras aeneus (17) \\
\hline & & Corydoras aeneus (2) & Crenicichla britskii (10) \\
\hline & & & Astyanax altiparanae (7) \\
\hline & & & Rhamdia quelen (4) \\
\hline
\end{tabular}

Tabela 6. Composição das guildas alimentares referente à comunidade de peixes do Córrego São Carlos ao longo do gradiente longitudinal. Entre parênteses está informado o número de indivíduos coletados. 


\begin{tabular}{lcccccccccc} 
& Aalti & Msanc & Opint & Rquel & Trich & Caene & Cbrits & Hison & Hanci & Hnigro \\
\hline Msanc & $0,95^{*}$ & - & - & - & - & - & - & - & - & - \\
Opint & $0,95^{*}$ & $0,99^{*}$ & - & - & - & - & - & - & - & - \\
Rquel & 0,46 & 0,52 & 0,48 & - & - & - & - & - & - & - \\
Trich & 0,30 & 0,45 & 0,42 & $0,90^{*}$ & - & - & - & - & - & - \\
Caene & 0,40 & 0,52 & 0,50 & $0,77^{*}$ & $0,78^{*}$ & - & - & - & - & - \\
Cbrits & 0,52 & $0,63^{*}$ & $0,61^{*}$ & $0,96^{*}$ & $0,95^{*}$ & $0,82^{*}$ & - & - & - & - \\
Hison & 0,06 & 0 & 0 & 0,08 & 0 & 0 & 0,03 & - & - & - \\
Hanci & 0 & 0 & 0 & 0 & 0 & 0 & 0 & $0,95^{*}$ & - & - \\
Hnigro & 0 & 0 & 0 & 0 & 0 & 0 & 0 & $0,96 *$ & $0,99 *$ & - \\
Pcaudi & 0,10 & 0,14 & 0,13 & 0,31 & 0,31 & 0,28 & 0,32 & $0,88^{*}$ & $0,86^{*}$ & $0,88^{*}$ \\
\hline
\end{tabular}

Tabela 7. Valores de sobreposição alimentar entre as espécies de peixes do Córrego São Carlos, Parque Estadual Morro do Diabo, sudeste do Brasil. Valores $\geq 0,58$ são considerados significativos (indicados por *). Aalti (Astyanax altiparanae), Msanc (Moenkhausia sanctaefilomenae), Opint (Oligosarcus pintoi), Rquel (Rhamdia quelen), Trich (Trichomycterus sp.), Caene (Corydoras aeneus), Cbrits (Crenicichla britskii), Hison (Hisonotus sp.), Hanci (Hypostomus ancistroides), Hnigro (Hypostomus nigromaculatus).

dieta de $P$. caudimaculatus também está refletida na sobreposição alimentar apresentada entre esta espécie e as perifitívoras. A captura do alimento se dá durante o dia, em remansos rasos, junto às margens, onde grupos de 5-15 indivíduos nadam ativamente contra a correnteza, próximos à superfície, catando pequenos itens arrastados pela corrente e na superfície (“drift-feeding” cf. Grant \& Noakes, 1987 e “surface picking”, cf. Sazima, 1986).

\subsubsection{Perifitívoros}

As três espécies de cascudos mostraram elevada sobreposição alimentar e ingeriram quase que exclusivamente perifíton. Todas utilizam a tática de pastejo ("grazing”, cf. Keenleyside, 1979) na qual os peixes ficam apoiados sobre rochas, troncos e vegetais submersos, de onde raspam a matriz perifítica. Nos estômagos analisados, algas apresentaram maior freqüência de ocorrência, dentre as quais se destacam as diatomáceas bentônicas (principalmente Tabellaria, Navicula, Pinnularia e Bacillaria) e clorofíceas (principalmente Spirogyra, Oedogonium e Mesotaenium).

No Córrego São Carlos, Hisonotus sp. forrageia durante o dia. Essa espécie utiliza as nadadeiras pares para manter sua estabilidade quando está aderida a folhas, galhos ou raízes submersas. Enquanto raspa com a boca, faz breves interrupções para se deslocar por curtas distâncias, geralmente de uma folha à outra. Hypostomus nigromaculatus forrageia ativamente a partir da metade do dia até o início da noite, em áreas mais correntosas entre saibros e cascalhos, deixando a parte posterior do corpo ondular pela correnteza. Hypostomus ancistroides começa a atividade de forrageio com o início da noite, principalmente junto às margens, sobre galhos e troncos submersos, onde os indivíduos ficam aderidos pela boca enquanto raspam, apresentando as nadadeiras peitorais distendidas o que, provavelmente, auxilia na sua estabilidade. Enquanto raspam, os indivíduos de $H$. ancistroides e $H$. nigromaculatus camuflam sobre o substrato, por homocromia.

\section{Discussão}

A importância do aporte de material alóctone para a alimentação dos peixes de riachos é bem documentada na literatura (vide Saul, 1975; Angermeier \& Karr, 1984; LoweMcConnell, 1999; Sabino \& Castro, 1990; Henry et al., 1994; Sabino \& Zuanon, 1998; Castro, 1999). Apesar de alguns trabalhos (Costa, 1978; Moyle \& Senanayake, 1984; Uieda et al., 1997 e presente estudo) registrarem a maior participação de itens autóctones nos estômagos (algas e invertebrados aquáticos), de fato estes itens são dependentes de nutrientes advindos da matéria orgânica

http://www.biotaneotropica.org.br 
carreada da vegetação ripária, considerada a base da cadeia trófica em ecossistemas de riachos (Gregory et al., 1991), o que acentua a importância da conservação de áreas ripárias para as comunidades aquáticas (Angermeier \& Karr, 1984).

Representantes das três guildas alimentares foram encontrados em todos os trechos estudados do Córrego São Carlos (vide Tabela 6). Dentre os perifitívoros, $H$. nigromaculatus apresenta constância decrescente ao longo do riacho e, dentre os invertívoros, o mesmo foi constatado para Trichomycterus sp. Essas duas espécies, de hábitos reofílicos, possuem micro-hábitats bastante específicos e ocorrem quase que exclusivamente em corredeiras que, por sua vez, são progressivamente mais raras em sentido jusante. As demais espécies de perifitívoros e invertívoros apresentam constância crescente ao longo do riacho provavelmente em função da adição longitudinal de microhábitats e, portanto, da adição de sítios disponíveis para alimentação, favorecendo espécies que exploram ativamente a coluna d’água (p. ex. A. altiparanae e $M$. sanctaefilomenae) e outras que exploram substratos arenosos (C. aeneus) e poços marginais (C. britskii). Esses resultados apontam para uma correlação positiva entre heterogeneidade de hábitat, diversidade de peixes e complexidade de suas relações (cf. Schlosser, 1982).

No Córrego São Carlos, 33\% dos pares de espécies utilizam recursos alimentares semelhantes, porém essa sobreposição não necessariamente invoca a existência de competição por alimento, podendo ser reflexo tanto da disponibilidade destes recursos (Hurlbert, 1978), que são diferencialmente partilhados (Ross, 1986), quanto do grau de inclusividade taxonômica de cada categoria alimentar utilizada na análise. No Brasil, estudos que envolvem a biologia alimentar de peixes de riachos (p. ex. Sabino \& Castro, 1990; Aranha et al., 1993; Buck \& Sazima, 1995; Uieda et al., 1997; Aranha et al., 1998; Casatti \& Castro, 1998) exemplificam casos em que ocorre partilha de recursos, apesar da existência de alguma sobreposição alimentar. Em adição, a inferência sobre a existência de sobreposição alimentar através de métodos indiretos deve considerar que a resolução taxonômica alcançada na identificação dos itens alimentares pode ser insuficiente para esclarecer como se caracterizam as presas ingeridas em termos de distribuição espacial e temporal. Este cuidado certamente influencia a interpretação de como ocorre a partilha de recursos e a estruturação das comunidades (vide Longenecker, 2001).

Apesar da semelhança na dieta das espécies invertívoras, a combinação de diferentes micro-hábitats, períodos de atividade e táticas utilizadas na captura do alimento certamente minimiza o efeito da sobreposição alimentar e representa uma situação comumente encontrada em riachos tropicais (vide Moyle \& Senanayake, 1984; Sabino \& Castro, 1990; Sabino \& Zuanon, 1998; Aranha et al., 1998; Casatti \& Castro, 1998).
A única espécie onívora, $P$. caudimaculatus, foi comum aos três trechos. Essa ampla distribuição ao longo do riacho estudado pode estar associada à sua flexibilidade alimentar, visto que essa espécie foi a que apresentou maior variabilidade de itens alimentares em termos de categorias ecológicas. Apesar de ter sido registrada apenas a cata de itens arrastados pela correnteza, a presença de fragmentos de vegetais superiores em 57,5\% dos estômagos analisados indica que esta espécie também pode praticar a poda, conforme Sabino \& Castro (1990) observaram em um riacho litorâneo do Brasil. Além disso, a elevada freqüência de diatomáceas bentônicas nos estômagos analisados pode também indicar a captura do alimento junto ao fundo.

Dentre os cascudos o período de forrageamento e os micro-hábitats explorados se combinam de forma distinta para cada uma das três espécies. Provavelmente o formato mais hidrodinâmico de $H$. nigromaculatus favorece o forrageamento em áreas mais correntosas, o que se reflete no fato desta espécie ser uma das mais bem sucedidas no trecho superior. Semelhante segregação espacial foi verificada por outros autores entre Microlepidogaster sp., Hypostomus garmani e Harttia sp. no Alto rio São Francisco (Casatti \& Castro, 1998); entre Ancistrus sp., Harttia kronei, Kronichthys subteres e Schizolecis guntheri em um riacho de Mata Atlântica (Buck \& Sazima, 1995) e entre Hypostomus sp., Microlepidogaster sp. e H. ancistroides em um riacho da bacia do Alto rio Paraná (Uieda et al., 1997). No caso específico da comunidade de pastadores perifitívoros, a evolução deste padrão de exploração espacial diferencial parece também diluir o impacto da predação sobre a comunidade de algas, apesar deste ser um recurso relativamente abundante em riachos (Uieda et al., 1997).

Assim, a comunidade de peixes no Córrego São Carlos mostra-se estruturada em nível espacial, temporal e trófico, apresentando uso partilhado dos recursos alimentares disponíveis. Se existe competição interespecífica por alimento, essa interação não foi detectada. Além disso, o acréscimo de espécies em cada categoria trófica ao longo do riacho possivelmente é um reflexo da crescente heterogeneidade longitudinal de micro-hábitats na área, disponibilizando sítios de alimentação adicionais (cf. Schlosser, 1982; Angermeier \& Karr, 1984). Trabalhos dessa natureza em sítios-referência específicos permitem compreender como se estruturam as comunidades de peixes e fornecer importantes informações subsidiárias para estudos de impactos pontuais e restauração (Barbour et al., 1996).

\section{Agradecimentos}

Agradeço Hertz F. Santos, Katiane M. Ferreira, Luiz S. F. Martins, Renata Stopiglia e Humberto F. Mendes pelo auxílio no campo; Katiane M. Ferreira pelo auxílio com a identificação de algas; Alex L. A. Melo pelo auxílio com a 
identificação de vegetais; Ricardo M. C. Castro, Francisco Langeani, Flávio A. Bockmann e Marcelo R. Britto pelo auxílio com a identificação de espécies de peixes; Instituto Florestal-SP, IBAMA, Parque Estadual Morro do Diabo e Departamento de Biologia FFCLRP-USP pelo apoio durante a realização deste trabalho; Virgínia S. Uieda pela leitura crítica do manuscrito; Richard P. Vari pela revisão do Abstract. Este trabalho foi financiado pela Fundação de Amparo à Pesquisa do Estado de São Paulo (FAPESP) dentro do Programa BIOTA/FAPESP - O Instituto Virtual da Biodiversidade (www.biota.org.br) - através do Projeto Temático "Diversidade de peixes de riachos e cabeceiras da bacia do Alto rio Paraná no Estado de São Paulo, Brasil” (processos n 98/05072-8, 00/01919-8) e pelo Projeto PRONEX “Conhecimento, Conservação e Utilização Racional da Diversidade da Fauna de Peixes do Brasil” (FINEP/CNPq n ${ }^{\circ}$ 661058/1997-2). A autora recebe auxílio financeiro da FAPESP (processos $n^{\circ}$ 01/13340-7, 02/05996-2).

\section{Referências bibliográficas}

ANGERMEIER, P.L. \& KARR, J.R. 1984. Fish communities along environmental gradients in a system of tropical streams. Env. Biol. Fish. 9:117-135.

ARANHA, J.M.R., CARAMASCHI, E.P. \& CARAMASCHI, U. 1993. Ocupação espacial, alimentação e época reprodutiva de duas espécies de Corydoras Lacépède (Siluroidei, Callichthyidae) coexistentes no Rio Alambari (Botucatu, São Paulo). Revta bras. Zool. 10:453-466.

ARANHA, J.M.R., TAKEUTI, D.F. \& YOSHIMURA, T.M. 1998. Habitat use and food partitioning of the fishes in a coastal stream of Atlantic Forest, Brazil. Rev. Biol. Trop. 46:951-959.

BARBOUR, M.T., GERRITSEN, J., SNYDER, B.D. \& STRIBLING, J.B. 1999. Rapid bioassessment protocols for use in streams and wadeable rivers: periphyton, benthic macroinvertebrates and fish. Second edition. EPA 841-B-99-002. U. S. Environmental Protection Agency; Office of Water, Washington, D.C.

BOWEN, S.H. 1992. Quantitative description of the diet. In Fisheries techniques (L.A. Nielsen \& D.L. Johnson, eds.). American Fisheries Society, Bethesda, p. 325-336.

BUCK, S. \& SAZIMA, I. 1995. An assemblage of mailed catfishes (Loricariidae) in southeastern Brazil: distribution, activity, and feeding. Ichthyol. Explor. Freshwaters 6:325-332.

CASATTI, L. \& CASTRO, R.M.C. 1998. A fish community of the São Francisco River headwaters riffles, southeastern Brazil. Ichthyol. Explor. Freshwaters 9:229-242.

CASATTI, L., LANGEANI, F. \& CASTRO, R.M.C. 2001. Peixes de riacho do Parque Estadual Morro do Diabo, bacia do Alto rio Paraná, SP. Biota Neotropica 1:1-15.
CASTRO, R.M.C. 1999. Evolução da ictiofauna de riachos sul-americanos: padrões gerais e possíveis processos causais. In Ecologia de Peixes de Riachos: Estado Atual e Perspectivas (E.P. Caramaschi, R. Mazzoni, C.R.S.F. Bizerril, P.R. Peres-Neto, eds.). Oecologia Brasiliensis, v. VI, PPGE-UFRJ, Rio de Janeiro, p. 139-155

CLAUSET, L.R. 1999. Paisagem paulista: áreas protegidas. Empresa das Artes, São Paulo.

COSTA, W.J.E.M. 1987. Feeding habits of a fish community in a tropical coastal stream, rio Mato Grosso, Brazil. Stud. Neotrop. Fauna \& Environm. 22:145-153.

CURIO, E. 1976. The ethology of predation. Springer, Berlin. DAJOZ, R. 1983. Ecologia geral. Ed. Vozes, São Paulo.

ESTEVES, K.E. \& ARANHA, J.M.R. 1999. Ecologia trófica de peixes de riachos. In Ecologia de Peixes de Riachos: Estado Atual e Perspectivas (E.P. Caramaschi, R. Mazzoni, C.R.S.F. Bizerril, P.R. Peres-Neto, eds.). Oecologia Brasiliensis, v. VI, PPGE-UFRJ, Rio de Janeiro, p. $157-182$

GIBRAN, F.Z. \& CASTRO, R.M.C. 1999. Activity, feeding behaviour and diet of Ogcocephalus vespertilio in southern west Atlantic. J. Fish Biol. 55:588-595.

GRANT, J.W.A. \& NOAKES, D.L.G. 1987. A simple model of optimal territory size for drift-feeding fishes. Can. J. Zool. 65:270-276.

GREGORY, S.V., SWANSON, F.J., McKEE, W.A. \& CUMMINS, K.W. 1991. An ecosystem perspective of riparian zones. Bioscience 41:540-551.

HENRY, R., UIEDA, V.S., AFONSO, A.A.O. \& KIKUCHI, R.M. 1994. Input of allochthonous matter and structure of fauna in a Brazilian headstream. Verh. Internat. Verein. Limnol. 25:1866-1870.

HORN, H.S. 1966. Measurement of “overlap” in comparative ecological studies. Am. Nat. 100:419-424.

HUGHES, R. M. 1995. Defining acceptable biological status by comparing with reference conditions. In Biological assessment and criteria: tools for water resource planning and decision making (W.S. Davis \& T.P. Simon, eds.). CRC Press Inc., Florida, p. 31-47.

HURLBERT, S.H. 1978. The measurement of niche overlap and some relatives. Ecology 59:67-77.

HYNES, H.B.N. 1950. The food of fresh-water sticklebacks (Gasterosteus aculeatus and Pygosteus pungitius), with a review of methods used in studies of the food of fishes. J. Anim. Ecol. 19:36-57.

INVENTÁRIO FLORESTAL DOESTADODE SÃO PAULO. 2000. Instituto Florestal, Governo do Estado de São Paulo e Secretaria do Meio Ambiente, São Paulo.

KARR, J.R. 1981. Assessment of biotic integrity using fish communities. Fisheries 6:21-27. 
KEENLEYSIDE, M.H.A. 1979. Diversity and adaptation in fish behaviour. Springer, Berlin.

KNÖPELL, H.A. 1970. Food of Central Amazonian fishes: contribution to the nutrient ecology of Amazonian rain forest streams. Amazoniana 2:257-352.

LEHNER, P.N. 1998. Handbook of ethological methods. Garland STPM Press, London, 672 pp.

LINTON, L.R., DAVIES, R.W. \& WRONA, F.J. 1981. Resource utilization indices: an assessment. J. An. Ecol. 50:283292.

LONGENECKER, K. 2001. The role of food in the community structure of reef fishes. Abstracts $81^{\text {st }}$. Annual Meeting of the American Society of Ichthyologists \& Herpetologists, State College, EUA, p. 89.

LOWE-McCONNELL, R.H. 1999. Estudos ecológicos em comunidades de peixes tropicais (A.M. de A. Vazzoler, A.A. Agostinho \& P.T.M. Cunnhingham, trad.), EDUSP, São Paulo.

McALECEE, N., LAMBSHEAD, P.J.D., PATERSON, G.L.J. \& GAGE, J.G. 1997. BioDiversity Professional. Beta-Version. The Natural History Museum and The Scottish Association for Marine Sciences.

MOYLE, P.B. \& SENANAYAKE, F.R. 1984. Resource partitioning among the fishes of rainforest streams in Sri Lanka. J. Zool. Lond. 202:195-223.

NIMER, E. 1989. Climatologia do Brasil. Secretaria de Planejamento e Coordenação da Presidência da República e IBGE, Rio de Janeiro.

ROSS, S.T. 1986. Resource partitioning in fish assemblages: a review of field studies. Copeia 1986:352-388.

SABINO, J. 1999. Comportamento de peixes em riachos: métodos de estudo para uma abordagem naturalística. In Ecologia de Peixes de Riachos: Estado Atual e Perspectivas (E.P. Caramaschi, R. Mazzoni, C.R.S.F. Bizerril, P.R. Peres-Neto, eds.). Oecologia Brasiliensis, v. VI, PPGE-UFRJ, Rio de Janeiro, p. 183-208.

SABINO, J. \& CASTRO, R.M.C. 1990. Alimentação, período de atividade e distribuição espacial dos peixes de um riacho da floresta Atlântica (Sudeste do Brasil). Rev. Brasil. Biol. 50:23-36.

SABINO, J. \& ZUANON, J. 1998. A stream fish assemblage in Central Amazonia: distribution, activity patterns and feeding behavior. Ichthyol. Explor. Freswaters 8:201-210.

SAUL, W.G. 1975. An ecological study of fishes at a site in upper Amazonian Ecuador. Proc. Nat. Acad. Sci. Phila. 127:93-134.

SAZIMA, I. 1986. Similarities in feeding behaviour between some marine and freshwater fishes in two tropical communities. J. Fish. Biol. 29:53-65.
SCHLOSSER, I.J. 1982. Fish community structure and function along two habitat gradients in a headwater stream. Ecol. Monogr. 52:395-414.

SMOGOR, R.A. \& ANGERMEIER, P.L. 1999. Relations between fish metrics and measures of anthropogenic disturbance in three IBI regions in Virginia. In Assessing the sustainability and biological integrity of water resources using fish communities (T.P. Simon, ed.). CRC Press, Boca Raton, Florida, p. 585-610.

UIEDA, V.S., BUZZATO, P. \& KIKUCHI, R.M. 1997. Partilha de recursos alimentares em peixes em um riacho de serra no sudeste do Brasil. An. Acad. Bras. Ci. 69:243-252.

VALENTIN, J.L. 1995. Agrupamento e ordenação. In Tópicos em tratamentos de dados biológicos (P.R. Peres-Neto, J.L. Valentin \& F.A.S. Fernandez, eds.). Oecologia Brasiliensis, v. II, PPGE-UFRJ, Rio de Janeiro, p. 25-55.

Título: Alimentação dos peixes em um riacho do Parque Estadual Morro do Diabo, bacia do Alto Rio Paraná, sudeste do Brasil.

Autora: Lilian Casatti

Biota Neotropica, Vol. 2, number 2: 2003

http://www.biotaneotropica.org.br/v2n2/pt/ a b stract ? a rticle + B N 02502022002

\section{ISSN 1676-0603}

Recebido em 02/09/2002

Revisado em 14/11/2002

Publicado em 23/11/2002 